Chronic Obstructive Pulmonary Diseases: Journal of the COPD Foundation

\author{
Original Research
}

\title{
Prevalence of Low Peak Inspiratory Flow Rate at Discharge in Patients Hospitalized for COPD Exacerbation
}

\author{
Gulshan Sharma, MD, MPH ${ }^{1}$ Donald A. Mahler, MD, FCCP ${ }^{2}$ Valerie M. Mayorga, PharmD ${ }^{3}$ \\ Kathleen L. Deering, PharmD ${ }^{3}$ Qing Harshaw, $\mathrm{MD}, \mathrm{PhD}^{3}$ Vaidyanathan Ganapathy, $\mathrm{PhD}^{4}$
}

\begin{abstract}
Background: Low peak inspiratory flow rate (PIFR) ( $<60 \mathrm{~L} / \mathrm{min}$ ) among patients with chronic obstructive pulmonary disease (COPD) may result in ineffective medication inhalation, leading to poor bronchodilation.

Objective: The objectives of this analysis were to evaluate the prevalence of low PIFR at the time of discharge from a COPD-related hospitalization and to examine the real-world treatment patterns and rehospitalizations by PIFR. Methods: Patients at 7 sites in the United States were screened for enrollment at hospital discharge. PIFR was measured using the InCheck ${ }^{\mathrm{TM}}$ DIAL to simulate resistance of the DISKUS ${ }^{\circledast}$ dry powder inhaler (DPI). An equal number of patients were enrolled into low PIFR ( $<60 \mathrm{~L} / \mathrm{min})$ or normal PIFR $(\geq 60 \mathrm{~L} / \mathrm{min})$ cohorts. Demographics, COPD-related clinical characteristics, health status, treatment and rehospitalization data were collected.

Results: Mean PIFR was $71 \pm 22.12 \mathrm{~L} / \mathrm{min}$ among 268 screened patients; $31.7 \%$ ( $\mathrm{n}=85$ ) of patients had low PIFR. Among all enrolled patients $(\mathrm{n}=170)$, the low PIFR cohort was older $(66.2 \pm 10.04$ years versus $62.1 \pm 9.41$ years, $p=0.006)$ and more likely to be female (61.2\% versus $42.4 \%, p=0.014)$. There was an increase in DPI use at discharge, compared with admission, in the low PIFR cohort (62.4\% versus $70.6 \%, p=0.020)$. The incidences of allcause rehospitalization up to 180 days were similar between the low and normal PIFR cohorts.

Conclusions: At discharge following hospitalization for an exacerbation of COPD, approximately one-third of patients had a PIFR <60 L/min. More patients with a low PIFR were discharged with a DPI medication compared with use at admission. There was no difference in the rehospitalization rates by PIFR.
\end{abstract}

Abbreviations: peak inspiratory flow rate, PIFR; chronic obstructive pulmonary disease, COPD; dry powder inhaler, DPI; metered dose inhalers, MDI; soft mist inhalers, SMI; modified Medical Research Council, mMRC; COPD Assessment Test, CAT; Morisky Medication Adherence Scale, MMAS; forced expiratory volume in 1 second, FEV $\mathbf{1}$; forced vital capacity, FVC; body mass index, BMI; length of stay, LOS; short-acting beta-agonist, SABA; short-acting muscarinic antagonist, SAMA; long-acting muscarinic antagonist, LAMA; long-acting beta2agonist, LABA; inhaled corticosteroids, ICS

Funding Support: Funding for this project was provided by Sunovion Pharmaceuticals, Inc.

Date of Acceptance: April 21, 2017

Citation: Sharma G, Mahler DA, Mayorga VM, Deering KL, Harshaw Q, Ganapathy V. Prevalence of low peak inspiratory flow rate at discharge in patients hospitalized for COPD exacerbation. Chronic Obstr Pulm Dis. 2017;4(3):217-224. doi: https://doi.org/10.15326/ jcopdf.4.3.2017.0183

1 University of Texas Medical Branch, Galveston, Texas

2 Geisel School of Medicine at Dartmouth, Hanover, New Hampshire and Valley Regional Hospital, Claremont, New Hampshire

3 EPI-Q, Inc., Oak Brook, Illinois

4 Sunovion Pharmaceuticals, Inc, Marlborough, Massachusetts

\section{Address correspondence to:}

Gulshan Sharma, MD, MPH

Professor and Director, Division of Pulmonary Critical Care \& Sleep Medicine

University of Texas Medical Branch

301 University Blvd

Galveston, TX 77555

Telephone: 409-772-2436

Email: gusharma@utmb.edu 


\section{Keywords:}

chronic obstructive pulmonary disease; COPD; discharge planning; exacerbation; outcomes research; peak inspiratory flow rate; PIFR

\section{Introduction}

Chronic lower respiratory disease, primarily chronic obstructive pulmonary disease (COPD), was the third leading cause of death in the United States in 2013, claiming the lives of 149,205 Americans. ${ }^{1}$ COPD treatment involves the use of both short- and longacting beta2-agonists and, more recently, muscarinic antagonists that are delivered through devices such as metered-dose inhalers (MDIs), dry powder inhalers (DPIs), soft mist inhalers (SMIs), and nebulizers. ${ }^{2}$ Each type of device has its own advantages and disadvantages in terms of delivery effectiveness versus motor coordination techniques. MDIs require complex coordination techniques with a slow inhalation by the patient to achieve a clinically effective dose., DPIs decrease the complexity of administration, but effective medication delivery is dependent on the force of the patient's inspiratory effort to overcome internal resistance. ${ }^{5}$ A relatively recent addition to the COPD market, SMIs deliver medication in a slow-moving mist independent of the patient's inspiratory effort and are less dependent on inhalation technique. ${ }^{6}$ Nebulizers are machines that deliver medication in a way that is independent of inspiratory effort and inhalation technique. ${ }^{7}$

DPIs have internal resistance and some patients may not be able to inhale an optimal amount of powder deep into the lower respiratory tract. Peak inspiratory flow rate (PIFR), the measure of a patient's inspiratory effort, can be used to assess a patient's ability to generate adequate inspiratory flow rate from DPIs such as the DISKUS ${ }^{\circledR}$. The literature suggests that a PIFR below $60 \mathrm{~L} / \mathrm{min}$ in COPD patients may result in ineffective inhalation of medications. ${ }^{8-10}$ We hypothesize that ineffective inhalation of medications due to low PIFR can result in poor COPD management and adverse consequences for the patient. Given that COPD generally affects an older, multi-morbid population, the mode of medication delivery may, therefore, be an important consideration for clinicians when selecting a treatment, especially upon discharge for a COPD exacerbation.

The prevalence of low PIFR in patients being discharged for a COPD exacerbation has not been studied to date. Generally, discharge protocols for
COPD patients do not include an assessment of PIFR or patients' ability to use their inhaler device when they recuperate after discharge. The objectives of this analysis were to evaluate the prevalence of and factors associated with low PIFR, the real-world treatment patterns by PIFR levels, and rehospitalizations among COPD patients at discharge from the hospital following an exacerbation.

\section{Methods}

This was an institutional review board-approved prospective, observational study of patients hospitalized for a COPD exacerbation at 7 sites in the United States. Patients were identified and screened for enrollment if they had a primary or secondary COPD diagnosis for the admission in their medical records and had a diagnosis of COPD for at least 1 year. All screened patients provided a PIFR value, which was measured using the InCheck ${ }^{\mathrm{TM}}$ DIAL, a device that has been widely used in previous studies. ${ }^{8,11-15}$ According to the manufacturer, the InCheck ${ }^{\mathrm{TM}}$ DIAL is accurate to +/$10 \%$ or $10 \mathrm{~L} / \mathrm{min}$, whichever is greater, and can measure flows in the range of 15 to $120 \mathrm{~L} / \mathrm{min}^{16}$ The InCheck $^{\mathrm{TM}}$ DIAL was set to simulate resistance of the DISKUS ${ }^{\circledR}$ DPI, a commonly used DPI device and has a lower resistance than the Handihaler ${ }^{\circledast}$ and Turbuhaler ${ }^{\circledast}$. ${ }^{12,17}$ Patients made 3 PIFR attempts, and study coordinators instructed each patient to inhale as quickly as possible with the InCheck ${ }^{\mathrm{TM}}$ DIAL after a complete exhalation.

The highest PIFR value for each enrolled patient was recorded and used for analysis and classification $^{8,11,12,13}$ into the low PIFR ( $<60 \mathrm{~L} / \mathrm{min}$ ) or normal PIFR ( $\geq 60 \mathrm{~L} / \mathrm{min}$ ) cohorts. A cutoff of $60 \mathrm{~L} /$ min was selected between the low and normal cohorts because the literature suggests that the optimal PIFR against the internal resistance of a DPI is at least 60 $\mathrm{L} / \mathrm{min}^{8-10}$ To maintain the observational nature of the study, PIFR measurement was not shared with the treating provider. Patients were recruited for enrollment between May 19, 2015 and March 1, 2016. Cohort enrollment was actively managed to achieve equal numbers of patients in the low and normal PIFR cohorts.

Demographics, clinical characteristics, COPD-related health status, and treatment data were collected for the patients who were enrolled in the study. The level of activity limitation or disability due to dyspnea was assessed with the modified Medical Research Council (mMRC) dyspnea scale, and the COPD-related health 
status and symptom burden was assessed with the COPD Assessment Test (CAT) score for the patients who were enrolled in the study. The mMRC dyspnea scale and the CAT score were selected because they are recommended in the Global initiative for chronic Obstructive Lung Disease (GOLD) strategies to define COPD symptom burden. ${ }^{2}$ The Morisky Medication Adherence Scale (MMAS), a widely used self-reported measure of adherence, was also collected for the enrolled patients. Scores on the MMAS range from 0 to 4, with a score of zero reflecting high adherence, scores of 1 and 2 reflecting medium adherence, and scores of 3 and 4 reflecting low adherence. All baseline data, including PIFR, were collected by study coordinators within the 24 hours prior to the discharge date.

Rehospitalization data to the index hospital system were collected via chart review for the 30, 31-90 and 180 days following index hospitalization.

Descriptive analysis was performed according to the study objectives. Prevalence of low PIFR ( $<60 \mathrm{~L} / \mathrm{min}$ ) was calculated using data from all patients screened for enrollment. Differences between low and normal PIFR cohorts were tested using chi-squared tests for proportions and Student's t-tests for continuous measures. McNemar's test was used to examine the change in patients' medication use between admission and discharge. Multiple logistic regression models were used to examine the predictors of low PIFR in the total patient population and in the subgroup of patients with pulmonary function tests. A $p$ value of $\leq 0.05$ was considered statistically significant. All analyses were performed using SAS version 9.2 (SAS Institute, Cary, North Carolina).

\section{Results}

A total of 268 patients were screened for enrollment. To achieve equal cohorts, 98 patients with normal PIFR ( $\geq 60 \mathrm{~L} / \mathrm{min}$ ) were excluded from further analysis after PIFR data were collected. A total of 170 patients were enrolled, with 85 patients enrolled in both the low and normal PIFR cohorts.

The mean PIFR for the 268 patients screened for enrollment was $71 \pm 22.12 \mathrm{~L} / \mathrm{min}$. Low PIFR $(<60 / \mathrm{min})$ was observed in $31.7 \%$ (85/268) of patients (Figure 1). Of the 85 patients with low PIFR, 15 (18\%) had a PIFR less than $40 \mathrm{~L} / \mathrm{min}, 23$ patients (27\%) had a PIFR between 40-49 L/min, and 47 (55\%) had a PIFR between $50-59 \mathrm{~L} / \mathrm{min}$.

\section{Figure 1. PIFR Distribution at Hospital Discharge}

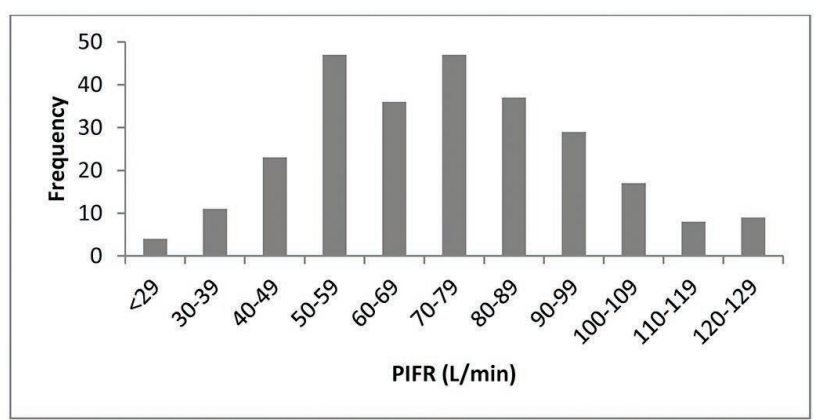

Distribution of PIFR (peak inspiratory flow rate) against simulated resistance of the DISKUS ${ }^{\circledR}$ dry powder inhaler at hospital discharge.

Demographic characteristics, clinical characteristics, and COPD-related health status data for the enrolled patients $(n=170)$ is presented in Table 1 . Among all enrolled patients, those in the low PIFR cohort were older $(66.2 \pm 10.04$ years versus $62.1 \pm 9.41$ years, $p=0.006)$ and were more likely to be women (61.2\% versus $42.4 \%, p=0.014)$ compared with patients with normal PIFR. Mean height was similar between patients in the low and normal PIFR cohorts (65.6 \pm 3.85 versus $66.4 \pm 3.99$ inches, $p=0.193$ ) and there was no difference in the height of women between the low and normal PIFR cohorts (63.7 \pm 3.00 versus $64.2 \pm 4.01$ inches, $p=0.496$ ). Based on the most recent pulmonary function tests, the mean forced expiratory volume in 1 second $\left(\mathrm{FEV}_{1}\right)$ percent predicted was between $30 \%$ and $49 \%$ of the normal range, suggesting severe disease.

There was no statistically significant difference in mMRC dyspnea score or CAT score between the low and normal PIFR cohorts. The mean CAT scores of patients were above 20, suggesting a high impact of COPD on the patients' health status, regardless of the PIFR (Table 1). Over $90 \%$ of patients in both the low and normal PIFR cohorts had a total CAT score $\geq 10$, the cutoff for higher symptom burden in the GOLD strategies. ${ }^{2}$ The mean mMRC dyspnea scores indicated that patients were symptomatic with daily activities in both PIFR cohorts. Over $80 \%$ of patients in both the low and normal PIFR cohorts had a total mMRC dyspnea score $\geq 2$, the cutoff for higher symptom burden in the GOLD strategies. ${ }^{2}$ No statistically significant correlation was found between PIFR and CAT score $(p=0.536)$ and PIFR and mMRC dyspnea 


\section{Table 1. Patient Baseline Demographics and Clinical Characteristics by Peak Inspiratory Flow Rate ${ }^{a}$}

\begin{tabular}{|c|c|c|c|c|}
\hline & $\begin{array}{c}\text { Total } \\
N=170\end{array}$ & $\begin{array}{c}<60 \text { L/min } \\
n=85\end{array}$ & $\begin{array}{c}\geq 60 \mathrm{~L} / \mathrm{min} \\
\quad n=85\end{array}$ & $P$ Value \\
\hline \multicolumn{5}{|l|}{ Demographic, mean ( \pm SD) } \\
\hline Age & $64.1 \pm 9.92$ & $66.2 \pm 10.04$ & $62.1 \pm 9.41$ & 0.006 \\
\hline Female, n (\%) & $88(51.8)$ & $52(61.2)$ & $36(42.4)$ & 0.014 \\
\hline \multicolumn{5}{|l|}{ Race, n (\%) } \\
\hline Caucasian, n (\%) & 78 (45.9) & $40(47.1)$ & $38(44.7)$ & 0.758 \\
\hline Black or African Heritage, n (\%) & $92(54.1)$ & $45(52.9)$ & $47(55.3)$ & \\
\hline Height (inches) & $66.0 \pm 3.93$ & $65.6 \pm 3.85$ & $66.4 \pm 3.99$ & 0.193 \\
\hline Weight (kgs) & $82.1 \pm 27.37$ & $83.2 \pm 28.89$ & $81.1 \pm 25.90$ & 0.618 \\
\hline BMI $\left(\mathrm{kg} / \mathrm{m}^{2}\right)$ & $29.1 \pm 9.13$ & $29.8 \pm 9.83$ & $28.3 \pm 8.37$ & 0.294 \\
\hline \multicolumn{5}{|l|}{ Smoking status } \\
\hline Current, n (\%) & $73(42.9)$ & $40(47.1)$ & $33(38.8)$ & 0.536 \\
\hline Former, n (\%) & $85(50.0)$ & $39(45.9)$ & $46(54.1)$ & \\
\hline Never, $\mathrm{n}(\%)$ & $12(7.1)$ & $6(7.1)$ & $6(7.1)$ & \\
\hline Years of COPD Diagnosis & $7.1 \pm 6.05$ & $7.9 \pm 6.80$ & $6.2 \pm 5.09$ & 0.068 \\
\hline LOS of Index Hospitalization & $4.4 \pm 3.23$ & $4.4 \pm 3.35$ & $4.4 \pm 3.13$ & 0.906 \\
\hline \multicolumn{5}{|l|}{ Pulmonary Function Tests ${ }^{b, c}$} \\
\hline FVC (L) & $2.3 \pm 0.87(n=68)$ & $2.2 \pm 0.78(n=32)$ & $2.4 \pm 0.94(n=36)$ & 0.307 \\
\hline FVC, $\%$ of predicted & $69.4 \% \pm 20.02(n=68)$ & $66.8 \% \pm 19.68(n=32)$ & $71.7 \% \pm 20.31(n=36)$ & 0.325 \\
\hline $\mathrm{FEV}_{1}(\mathrm{~L} / \mathrm{sec})$ & $1.2 \pm 0.58(\mathrm{n}=68)$ & $1.1 \pm 0.51(n=32)$ & $1.3 \pm 0.62(n=36)$ & 0.103 \\
\hline $\mathrm{FEV}_{1}, \%$ of predicted & $46.2 \% \pm 19.24(\mathrm{n}=68)$ & $42.2 \% \pm 18.70(n=32)$ & $49.7 \% \pm 19.29(n=36)$ & 0.110 \\
\hline $\mathrm{FEV}_{1} / \mathrm{FVC}$ ratio $\mathrm{x} 100$ & $52 \pm 15(\mathrm{n}=68)$ & $49 \pm 16(n=32)$ & $54 \pm 14(n=36)$ & 0.214 \\
\hline Total CAT Score & $25.2 \pm 8.10$ & $24.6 \pm 8.45$ & $25.8 \pm 7.74$ & 0.316 \\
\hline Total mMRC Score & $2.8 \pm 1.22$ & $2.9 \pm 1.26$ & $2.7 \pm 1.20$ & 0.533 \\
\hline Total MMAS Score & $1.0 \pm 1.17$ & $1.0 \pm 1.16^{\mathrm{d}}$ & $1.1 \pm 1.18$ & 0.601 \\
\hline
\end{tabular}

${ }^{a}$ PIFR is against simulated resistance of the Diskus ${ }^{\circledR}$ dry powder inhaler

${ }^{b}$ Most recent pulmonary function tests, as available.

${ }^{\mathrm{c}}$ Predicted values for pulmonary function tests based on NHANES III (Hankinson).

${ }^{\mathrm{d}}$ One patient in the $<60 \mathrm{~L} / \mathrm{min}$ cohort did not have MMAS score because they were on no medications prior to admission.

$\mathrm{BMI}=$ body mass index; $\mathrm{COPD}=$ chronic obstructive pulmonary disease; LOS=length of stay; $\mathrm{FVC}=$ forced vital capacity; $\mathrm{FEV}$ =forced expiratory volume in 1 second; CAT $=$ COPD Assessment Test; mMRC=modified Medical Research Council; MMAS=Morisky Medication Adherence Scale; PIFR=peak inspiratory flow rate.

score $(p=0.148)$. The mean MMAS score indicated medium medication adherence in both PIFR cohorts, and there was no difference between the cohorts. Multiple logistic regression analysis was performed to predict low PIFR with patient demographics and disease severity measures as predictors. Compared to men, women showed a greater chance of having low PIFR (odds ratio $=2.411, p=0.0457$ ). As age increased, the chance to have low PIFR also increased (odds ratio $=1.052, p=0.0058$ ). Compared to nonsmokers, the chance to have low PIFR was twofold higher for current smokers (odds ratio=2.055, $p=0.0422$ ).

In the analysis of comorbidities at enrollment (Table
2), pneumonia was a more common comorbidity among patients in the low PIFR cohort (38.8\% versus $22.4 \%, p=0.020$ ). There were more patients in the low PIFR cohort with a current diagnosis of pneumonia $(10.6 \%$ versus $2.4 \%, p=0.029)$ and there was a trend towards increased history of pneumonia in the low PIFR cohort (28.2\% versus $16.5 \%, p=0.066)$. Comorbid ischemic heart disease was also more common in the low PIFR cohort (14.1\% versus 3.5\%, $p=0.015)$.

Treatments at discharge following index hospitalization were similar to those at the time of admission in both cohorts (Table 3), with a few exceptions. As expected, the use of intravenous/oral 


\section{Table 2. Patient Baseline Comorbidities by Peak Inspiratory Flow Rate ${ }^{a}$}

\begin{tabular}{|c|c|c|c|c|}
\hline Comorbidities, n (\%) & $\begin{array}{c}\text { Total } \\
\mathbf{N}=170\end{array}$ & $\begin{array}{c}<60 \text { L/min } \\
n=85\end{array}$ & $\begin{array}{c}\geq 60 \mathrm{~L} / \mathrm{min} \\
n=85\end{array}$ & $P$ Value \\
\hline Congestive Heart Failure & $51(30.0)$ & $27(31.8)$ & $24(28.2)$ & 0.616 \\
\hline Ischemic Heart Disease & $15(8.8)$ & $12(14.1)$ & $3(3.5)$ & 0.015 \\
\hline Hypertension & $124(72.9)$ & $63(74.1)$ & $61(71.8)$ & 0.730 \\
\hline History of Myocardial Infarction & 31 (18.2) & $18(21.2)$ & $13(15.3)$ & 0.321 \\
\hline Atrial Fibrillation & $24(14.1)$ & $12(14.1)$ & $12(14.1)$ & 1.00 \\
\hline Diabetes & $63(37.1)$ & $29(34.1)$ & $34(40.0)$ & 0.427 \\
\hline Emphysema & $61(35.9)$ & 32 (37.6) & $29(34.1)$ & 0.631 \\
\hline Asthma & 67 (39.4) & $28(32.9)$ & $39(45.9)$ & 0.084 \\
\hline Bronchitis, Chronic & $44(25.9)$ & $23(27.1)$ & $21(24.7)$ & 0.726 \\
\hline Bronchiectasis & $3(1.8)$ & $1(1.2)$ & $2(2.4)$ & 0.560 \\
\hline Tuberculosis & $2(1.2)$ & 0 & $2(2.4)$ & 0.497 \\
\hline Other Respiratory Infections & $52(30.6)$ & $33(38.8)$ & $19(22.4)$ & 0.020 \\
\hline Pneumonia, current & $11(6.5)$ & 9 (10.6) & $2(2.4)$ & 0.029 \\
\hline Pneumonia, history of & $38(22.4)$ & $24(28.2)$ & $14(16.5)$ & 0.066 \\
\hline Pneumonia, history of and current & $2(1.2)$ & 0 & $2(2.4)$ & 0.497 \\
\hline Depression & $51(30.0)$ & $24(28.2)$ & $27(31.8)$ & 0.616 \\
\hline Anxiety & $54(31.8)$ & $28(32.9)$ & 26 (30.6) & 0.742 \\
\hline Osteoporosis & $10(5.9)$ & $6(7.1)$ & $4(4.7)$ & 0.515 \\
\hline Lung Cancer & $7(4.1)$ & $3(3.5)$ & $4(4.7)$ & 1.00 \\
\hline Other Cancer & 17 (10.0) & $6(7.1)$ & $11(12.9)$ & 0.201 \\
\hline
\end{tabular}

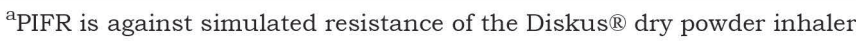

PIFR=peak inspiratory flow rate.

corticosteroid was higher at discharge compared with at admission in both cohorts (low PIFR: $28.2 \%$ versus $70.6 \%, p<0.0001$; normal PIFR: $32.9 \%$ versus $76.5 \%$, $p<0.0001$ ). The short-acting beta-agonist (SABA)/ short-acting muscarinic antagonist (SAMA) SMI use was lower at discharge compared with at admission in the low PIFR cohort (12.9\% versus $7.1 \%, p=0.025)$. More patients were discharged on a long-acting muscarinic antagonist (LAMA) DPI in both cohorts (low PIFR: $44.7 \%$ versus $51.8 \%$, $p=0.034$; normal PIFR: $42.4 \%$ versus $54.1 \%, p=0.012$ ). There was a trend towards increased long-acting beta2-agonist (LABA)/ inhaled corticosteroids (ICS) DPI use in the low PIFR cohort at discharge (42.4\% versus $49.4 \%, p=0.058$ ). There was increased DPI use in the low PIFR cohort at discharge, with $62.4 \%$ of patients in the low PIFR cohort receiving bronchodilator medications via a DPI device at admission and $70.6 \%$ at discharge $(p=0.020)$. More than half of the patients in both the low and normal PIFR cohorts were discharged with a nebulized therapy (60.0\% and $52.9 \%$, respectively).

All-cause rehospitalizations were similar between the low and normal PIFR cohorts during the follow-up period (Table 4). The incidences of all-cause rehospitalization at $30,31-90$ and 180 days following index hospitalization were: $15.3 \%, 11.8 \%$ and $40 \%$ respectively in the low PIFR cohort and 16.5\%, $12.9 \%$ and $37.6 \%$ respectively in the normal PIFR cohort ( $p$ values for differences not significant for any time point).

\section{Discussion}

The mean PIFR at discharge following hospitalization for an exacerbation of COPD was $71 \pm 22.12 \mathrm{~L} / \mathrm{min}$, and $31.7 \%$ of patients had PIFR less than $60 \mathrm{~L} / \mathrm{min}$. To our knowledge, our results are the first to describe the prevalence of low PIFR in patients at discharge following hospitalization for an exacerbation of COPD. Prior studies $8,12,13$ have assessed PIFR in clinically stable patients with COPD. A COPD exacerbation may have a deleterious effect on peak inspiratory flow due to lung hyperinflation. Therefore, it is important to measure PIFR at discharge to determine whether the patient can adequately inhale dry powder from a DPI, if such medication is prescribed.

Our PIFR cohorts are based on the literature demonstrating a PIFR over $60 \mathrm{~L} / \mathrm{min}$ is considered optimal. $^{8,12,18}$ We found the prevalence of low PIFR $(<60 \mathrm{~L} / \mathrm{min})$ to be $31.7 \%$ following hospitalization for a COPD exacerbation. In a study in the outpatient setting of patients with advanced COPD, Mahler and colleagues ${ }^{13}$ found the prevalence of PIFR less than 60 $\mathrm{L} / \mathrm{min}$ to be $19 \%$. Additionally, we found patients in the low PIFR cohort following hospitalization for a COPD exacerbation to be older and more likely to be women, with no differences in height or pulmonary function tests between cohorts. Mahler et $\mathrm{al}^{13}$ found that the majority of patients with PIFR less than $60 \mathrm{~L} / \mathrm{min}$ were female, of shorter height, and with lower percent predicted forced vital capacity (FVC) and inspiratory capacity. The differences in enrolled patient populations and timing of PIFR measurement may account for the 


\section{Table 3. Admission and Discharge Medications by Peak Inspiratory Flow Rate ${ }^{a}$}

\begin{tabular}{|c|c|c|c|c|}
\hline & \multicolumn{2}{|c|}{ PIFR $<60 \mathrm{~L} / \mathrm{min}$} & \multicolumn{2}{|c|}{ PIFR $\geq 60 \mathrm{~L} / \mathrm{min}$} \\
\hline & $\begin{array}{c}\text { Admission } \\
\mathrm{n}(\%)\end{array}$ & $\begin{array}{c}\text { Discharge } \\
\mathrm{n}(\%)\end{array}$ & $\begin{array}{l}\text { Admission } \\
\mathrm{n}(\%)\end{array}$ & $\begin{array}{c}\text { Discharge } \\
n(\%)\end{array}$ \\
\hline Non-Nebulized/Handheld & 79 (92.9) & $78(91.8)$ & $76(89.4)$ & 80 (94.1) \\
\hline $\begin{array}{l}\text { SABA } \\
\text { MDI }\end{array}$ & 60 (70.6) & $59(69.4)$ & $59(69.4)$ & $59(69.4)$ \\
\hline $\begin{array}{r}\text { SAMA } \\
\text { MDI }\end{array}$ & $7(8.2)$ & $7(8.2)$ & $3(3.5)$ & $2(2.4)$ \\
\hline $\begin{array}{l}\text { SABA/SAMA } \\
\text { SMI }\end{array}$ & $11(12.9)^{\mathrm{b}}$ & $6(7.1)^{\mathrm{b}}$ & $5(5.9)$ & $3(3.5)$ \\
\hline $\begin{array}{l}\text { ICS } \\
\text { MDI } \\
\text { DPI }\end{array}$ & $\begin{array}{l}4(4.7) \\
3(3.5) \\
1(1.2)\end{array}$ & $\begin{array}{l}5(5.9) \\
3(3.5) \\
2(2.4)\end{array}$ & $\begin{array}{l}4(4.7) \\
3(3.5) \\
2(2.4)\end{array}$ & $\begin{array}{l}4(4.7) \\
2(2.4) \\
2(2.4)\end{array}$ \\
\hline $\begin{array}{r}\text { LABA } \\
\text { DPI }\end{array}$ & 0 & 0 & $1(1.2)$ & $1(1.2)$ \\
\hline $\begin{array}{l}\text { LAMA } \\
\text { DPI }\end{array}$ & $38(44.7)^{\mathrm{b}}$ & $44(51.8)^{\mathrm{b}}$ & $36(42.4)^{b}$ & $46(54.1)^{b}$ \\
\hline $\begin{array}{l}\text { LABA/LAMA } \\
\text { DPI }\end{array}$ & 0 & 0 & $1(1.2)$ & $1(1.2)$ \\
\hline $\begin{array}{l}\text { LABA/ICS } \\
\text { MDI } \\
\text { DPI } \\
\text { Fluticasone/salmeterol (Advair DISKUS }{ }^{\circledR} \text { ) }\end{array}$ & $\begin{array}{l}56(65.9) \\
20(23.5) \\
36(42.4) \\
35(41.2)\end{array}$ & $\begin{array}{l}59(69.4) \\
18(21.2) \\
42(49.4) \\
41(48.2)\end{array}$ & $\begin{array}{l}56(65.9) \\
27(31.8) \\
33(38.8) \\
32(37.7)\end{array}$ & $\begin{array}{l}60(70.6) \\
26(30.6) \\
38(44.7) \\
38(44.7)\end{array}$ \\
\hline Any DPI Use & $53(62.4)^{b}$ & $60(70.6)^{b}$ & $57(67.1)$ & $63(74.1)$ \\
\hline Any MDI Use & $68(80.0)$ & $67(78.8)$ & 64 (75.3) & 66 (77.7) \\
\hline $\begin{array}{l}\text { Nebulized } \\
\text { SABA } \\
\text { SABA/SAMA } \\
\text { LABA } \\
\quad \text { Arformoterol (Brovana }{ }^{(\mathbb{}} \text { ) }\end{array}$ & $\begin{array}{r}50 \text { (58.8) } \\
36(42.4) \\
19(22.4) \\
1(1.2) \\
0\end{array}$ & $\begin{array}{r}51 \text { (60.0) } \\
36(42.4) \\
20(23.5) \\
4(4.7) \\
3(3.5)\end{array}$ & $\begin{array}{r}45 \text { (52.9) } \\
35(41.2) \\
13(15.3) \\
1(1.2) \\
1(1.2)\end{array}$ & $\begin{array}{r}45 \text { (52.9) } \\
29(34.1) \\
19(22.4) \\
2(2.4) \\
2(2.4)\end{array}$ \\
\hline $\begin{array}{l}\text { Other therapies } \\
\text { SABA } \\
\text { Steroid } \\
\text { Methylxanthine } \\
\text { Oxygen }\end{array}$ & $\begin{array}{r}0 \\
24(28.2)^{\mathrm{b}} \\
3(3.5) \\
37(43.5)\end{array}$ & $\begin{array}{r}0 \\
60(70.6)^{\mathrm{b}} \\
3(3.5) \\
39(45.9)\end{array}$ & $\begin{array}{r}2(2.4) \\
28(32.9)^{\mathrm{b}} \\
1(1.2) \\
42(49.4)\end{array}$ & $\begin{array}{r}1(1.2) \\
65(76.5)^{\mathrm{b}} \\
2(2.4) \\
38(44.7)\end{array}$ \\
\hline
\end{tabular}

\footnotetext{
aPIFR is against simulated resistance of the DISKUS $R$ DPI.

${ }^{b} \mathrm{P}$ value $<0.05$ for comparison of proportions on medications at admission vs. discharge.

Note: Patients may be counted in multiple treatment groups. The numbers for the ICS, LABA/ICS, and nebulized treatment categories add up to greater than the total because patients were receiving multiple therapies (i.e., a patient on both a nebulized SABA and a nebulized LABA would count as " 1 " in the nebulized SABA row and " 1 " in the nebulized LABA row, but only count as "1" in the total nebulized row).

PIFR=peak inspiratory flow rate; $\mathrm{SABA}=$ short-acting beta agonist; $\mathrm{MDI}=$ metered dose inhaler; SAMA=short-acting muscarinic antagonist; SMI=soft mist inhaler; DPI=dry-powder inhaler; ICS=inhaled corticosteroid; LABA=long-acting beta agonist; LAMA=long-acting muscarinic antagonist.
}

different study results. Study findings suggested that pneumonia and ischemic heart disease were associated with low PIFR. The clinical rationale for these findings are not clear and could be a reflection of unobserved confounders due to low sample sizes.

Our data suggest that PIFR did not impact all-cause rehospitalizations up to the 180-day follow-up period. However, it should be noted that the study was not powered for these outcomes and there was only a small sample of patients with rehospitalizations. Additionally, the analysis did not control for medication use and hospitalizations outside the index hospital system. Further, large studies are needed for evaluating impact of PIFR on rehospitalization.

PIFR can be used to assess a patient's ability to generate adequate inspiratory flow rate, and thus can be used to guide COPD treatment choices. DPIs have internal resistance and patients with diminished PIFR may not be able to inhale medications using a DPI effectively into the lower respiratory tract. Despite 


\section{Table 4. Hospitalizations After Index Hospitalization by Peak Inspiratory Flow Rate ${ }^{a, b}$}

\begin{tabular}{|c|c|c|c|}
\hline Hospitalizations, n (\%) & $\begin{array}{l}\text { Total } \\
N=170\end{array}$ & $\begin{array}{c}<60 \mathrm{~L} / \mathrm{mir} \\
n=85\end{array}$ & $\begin{array}{l}\geq 60 \text { L/min P Value } \\
n=85\end{array}$ \\
\hline
\end{tabular}

Patients with an all-cause

$66(38.8)$

$34(40.0)$

32 (37.6)

0.753

hospitalization in 180 days

Patients with an all-cause

$27(15.9)$

$13(15.3)$

$14(16.5)$

0.834

hospitalization within 30 days

of index hospitalization

Patients with an all-cause

$21(12.4)$

$10(11.8)$

$11(12.9)$

0.816

hospitalization between 30-90

days of index hospitalization

${ }^{a}$ PIFR is against simulated resistance of the DISKUS ${ }^{\circledR}$ dry powder inhaler

${ }^{\mathrm{b}}$ Hospitalizations determined by chart review.

PIFR=peak inspiratory flow rate.

exacerbation to congestive heart failure exacerbation after discharge for a small number of patients. Due to the similarities in patient treatments, these patients were not excluded from further analysis. Finally, enrollment numbers were not consistent across study sites, and thus site-specific treatment patterns could have had an influence on our overall results.

In conclusion, approximately onethird of COPD patients had a low PIFR (<60 L/min) prior to discharge from hospitalization following an exacerbation of COPD. Patients in the low PIFR group were older and more likely to be female. Future research is needed to understand the optimal treatment of patients with low PIFR

the patient's inability to generate optimal inspiratory flow, nearly two-thirds of patients in the low PIFR cohort received bronchodilator medications via a DPI device at discharge for a COPD exacerbation. Existing discharge protocols for COPD patients typically do not include an assessment of PIFR at discharge for a COPD exacerbation. Prior to our study, the number of patients discharged who are unable to generate adequate peak inspiratory flow was unclear. Our study aimed to identify the prevalence of low PIFR in this patient population and highlight the use of DPIs at discharge in patients for whom these inhalation devices may not be effective.

There are a few limitations to note in our study. First, we measured PIFR only against the simulated resistance of the DISKUS ${ }^{\circledR}$ DPI. We chose the DISKUS $^{\circledR}$ DPI because it is a commonly used DPI device, and because the DISKUS ${ }^{\circledR}$ DPI has lower internal resistance than the Handihaler $^{\circledR}$ and Turbuhaler ${ }^{\circledR}$ DPIs. ${ }^{8,10,12}$ Second, sites used multiple coordinators administering the PIFR reading with the InCheck $^{\mathrm{TM}}$ DIAL. Site coordinators were trained on the use of the InCheck ${ }^{\mathrm{TM}}$ DIAL; however, there could be inter-observer differences in technique. Third, data recorded for tiotropium did not specify whether the inhaler used was a DPI or SMI. Upon verification with the study sites that the majority of tiotropium use was DPI, the decision was made to categorize this medication as a DPI for analysis. Fourth, the admission diagnosis was used to identify patients for this study, and may have been updated from COPD compared with patients with normal PIFR.

\section{Acknowledgments}

All named authors meet the International Committee of Medical Journal Editors (ICMJE) criteria for authorship for this manuscript by contributing to conception, design, or data process; drafting and critically revising the manuscript; and approving this draft for submission and accepting responsibility for the contents within. Authors wish to thank Claudette Knight, PharmD, of Percolation Communications for medical editorial assistance. Financial support for medical editorial assistance was provided by Sunovion Pharmaceuticals, Inc.

\section{Declaration of Interest}

Gulshan Sharma serves on the scientific advisory board at Sunovion Pharmaceuticals, Inc. Donald A. Mahler serves on an advisory board and a speaker's bureau for Sunovion Pharmaceuticals, Inc. Valerie M. Mayorga, Kathleen L. Deering, and Qing Harshaw are employed at EPI-Q, Inc., which received funding from Sunovion Pharmaceuticals, Inc., for this analysis. Vaidyanathan Ganapathy is a full-time employee of Sunovion Pharmaceuticals, Inc. The content of this manuscript, the ultimate interpretation, and the decision to submit for publication was made by the authors independently. 


\section{References}

1. Centers for Disease Control and Prevention. National Center for Health Statistics. Deaths: Final Data for 2013. Nat Vital Stat Rep.2016; 64 (2):1-119.

2. Global Initiative for Chronic Obstructive Lung Disease. Global Strategy for the Diagnosis, Management and Prevention of COPD, 2016. GOLD website. www.goldcopd.org. Published 2016. Accessed July 18, 2016.

3. Pauwels R, Newman S, Borgstrom L. Airway deposition and airway effects of antiasthma drugs delivered from metered-dose inhalers. Eur Respir J. 1997;10 (9): 2127-2138.

doi: https://doi.org/10.1183/09031936.97.10092127

4. Newman SP. Principles of metered-dose inhaler design. Respir Care. 2005;50(9):1177-1190.

5. Newman SP. Drug delivery to the lungs from dry powder inhalers. Curr Opin Pulm Med. 2003;9(suppl 1): S17-20. doi: https://doi.org/10.1097/00063198-200304001-00005

6. Anderson P. Use of Respimat ${ }^{\circledR}$ Soft MistTM inhaler in COPD patients. Int J Chron Obstruct Pulmon Dis. 2006;1(2):251-259. doi: https://doi.org/10.2147/copd.2006.1.3.251

7. Geller DE. Comparing clinical features of the nebulizer, metereddose inhaler, and dry powder inhaler. Respir Care. 2005;50 (10):1313-1321.

8. Janssens W, VandenBrande P, Hardeman E, et al. Inspiratory flow rates at different levels of resistance in elderly COPD patients. Eur Respir J. 2008;31(1):78-83. doi: https://doi.org/10.1183/09031936.00024807

9. Mahler DA, Waterman LA, Ward J, Gifford AH. Comparison of dry powder versus nebulized beta-agonist in patients with COPD who have suboptimal peak inspiratory flow rate. $J$ Aerosol Med Pulm Drug Deliv. 2014;27(2):103-109. doi: https://doi.org/10.1089/jamp.2013.1038

10. Al-Showair RA, Tarsin WY, Assi KH, Pearson SB, Chrystyn H. Can all patients with COPD use the correct inhalation flow with all inhalers and does training help? Respir Med. 2007;101 (11):2395-2401.

doi: https://doi.org/10.1016/j.rmed.2007.06.008

11. Jarvis S, Ind PW, Shiner RJ. Inhaled therapy in elderly COPD patients; time for re-evaluation? Age Ageing. 2007;36 (2):213218b. doi: https://doi.org/10.1093/ageing/afl174

12. van der Palen J. Peak inspiratory flow through Diskus and Turbuhaler, measured by means of a peak inspiratory flow meter (In-Check DIAL). Respir Med. 2003; 97(203):285-289.

doi: https://doi.org/10.1053/rmed.2003.1289

13. Mahler DA, Waterman LA, Gifford AH. Prevalence and COPD phenotype for a suboptimal peak inspiratory flow rate against the simulated resistance of the Diskus ${ }^{\circledR}$ dry powder inhaler. $J$
Aerosol Med Pulm Drug Deliv. 2013;26(3):174-179.

doi: https://doi.org/10.1089/jamp.2012.0987

14. Baba K, Tanaka H, Nishimura M, et al. Age-dependent deterioration of peak inspiratory flow with two kinds of dry powder corticosteroid inhalers (Diskus and Turbuhaler) and relationships with asthma control. J Aerosol Med Pulm Drug Deliv. 2011;24(6):293-301.

doi: https://doi.org/10.1089/jamp.2010.0868

15. Banno M, Ibata H, Niimi T, Sato S, Matsushita R. The usefulness of inspiratory flow rate during inhalation corticosteroid therapy in asthma. Respiration. 2009;78(4): 387-392. doi: https://doi.org/10.1159/000220097

16. Alliance Tech Medical, Inc. In-Check DIAL Inhaler Technique Training and Assessment Tool. Alliance Tech Medical website. http://alliancetechmedical.com/products/check-dial-trainingdevice/. Accessed July 18, 2016.

17. Dederichs J, Singh D, Pavkov R. Inspiratory flow profiles generated by patients with COPD through the Breezhaler ${ }^{\circledR}$ inhaler and other marketed dry powder inhalers. Am J Respir Crit Care Med. 191;2015: A5793.

18. Atkins PJ. Dry powder inhalers: an overview. Respir Care. 2005;50(10):1304-1312. 